\section{Mortalidad por cáncer oral en Chile, 2002-2012}

\author{
JORGE CANDIA ${ }^{1, \mathrm{a}}$, ALEJANDRA FERNÁNDEZ $^{1, \mathrm{a}, \mathrm{b}}$, \\ CAROLINA SOMARRIVA ${ }^{1, \mathrm{a}, \mathrm{b}}$, OLIVIA HORNA-CAMPOS ${ }^{2, \mathrm{c}}$
}

\section{Deaths due to oral cancer in Chile in the period 2002-2012}

Background: Oral cancer is the $15^{\text {th }}$ most common cause of cancer death in the world. In Chile, $1 \%$ of all cancer deaths are related to oral and pharyngeal cancer. Aim: To determine mortality rates for oral cancer in Chile and its regions between 2002-2012. Material and Methods: Deaths and their causes between the years 2002-2012 were obtained from the Chilean National Statistics Institute. Crude and adjusted rates by age and sex were calculated for the country and its regions. The denominator was Chilean population on June 30, 2012 and the WHO standard population. Results: In the period studied, 1,611 individuals with a mean age of 67.6 years $(63 \%$ men) died because of oral cancer. The most common location of the tumor was the tongue in $27 \%$ of cases and the parotid gland in 16\%. The adjusted mortality rate in Chile was 0.85 / 100,000 inhabitants (1.13 and 0.58 in men and women, respectively). The regions with the highest rates were Antofagasta (1.51), Aysén (1.22) and Magallanes (1.17). Deaths among men occurred at younger ages than women. Conclusions: Mortality rates due to oral cancer in Chile are lower than abroad. The highest rates observed in some regions may be influenced by environmental factors such as arsenic contamination in Antofagasta and the lack of specialists and specialized care centers in Aysén and Magallanes.

(Rev Med Chile 2018; 146: 487-493)

Key words: Epidemiology; Mortality; Mouth Neoplasms.

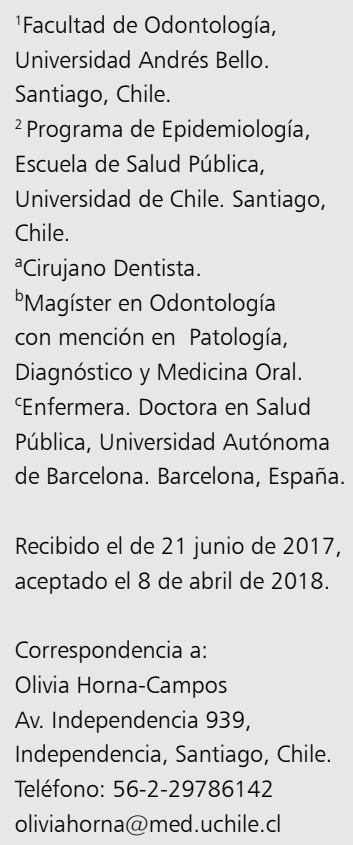

A nivel mundial, el cáncer oral (CO) es un serio problema de salud pública por el impacto y consecuencias que causa en la vida de los afectados y sus familiares ${ }^{1}$. Representa el $2 \%$ de todos los cánceres nuevos y es considerada la decimaquinta causa más común de muerte por cáncer en el mundo, con más de 145.353 casos y una tasa ajustada de 1,9 por 100.000 habitantes en 2012. Esta tasa es mayor en hombres en comparación con las mujeres $(2,7$ y 1,2 , respectivamente) y se distribuye de manera heterogénea según región, observándose una tasa de mortalidad de 2,2 por 100.000 habitantes (3,0 en hombres vs 1,4 mujeres) en Asia, en Europa 1,8 (3,0 hombres vs 0,7 mujeres) y en el Caribe y Sudamérica 1,2 (1,9 hombres vs 0,7 mujeres) $)^{2,3}$. Generalmente, las tasas más altas se han asociado a factores como mascar nuez de betel, consumo de tabaco, algunos hábitos alimentarios, como el consumo de carnes rojas, virus del papiloma humano y mala higiene dental, entre otras ${ }^{4}$.

La neoplasia maligna más frecuente de la cavidad oral es el carcinoma oral de células escamosas (COCE) y deriva del epitelio escamoso estratificado de la mucosa oral, con una prevalencia de 95\%, correspondiendo a 9 de cada 10 neoplasias orales y una supervivencia de $50 \%$ a los 5 años $^{5,6}$.

En Chile es escasa la información científica referente a la mortalidad por cáncer oral. En un estudio realizado por Riera et al. ${ }^{5}$, reportaron que 
la mortalidad por cáncer oral y faríngeo asciende a $1 \%$ del total de muertes por neoplasias malignas entre los años 1955 y 2002 . Por otro lado, Ramírez et al. ${ }^{7}$ reportaron que la mortalidad de cáncer oral y bucofaríngeo, entre los años 2002 y 2010, fue superior en hombres (1,69 por cada 100.000), en mayores de 55 años y la localización más frecuente fue cáncer en lengua.

En Chile, el Programa Nacional del Cáncer no cuenta con un programa específico para el cáncer oral y tampoco es parte de las garantías explícitas en salud del Ministerio de Salud (MINSAL), lo que reduce las posibilidades de acceso al diagnóstico y tratamiento oportuno en la gran mayoría de los pacientes ${ }^{8}$. Por ello, el objetivo del estudio es determinar la tasa de mortalidad para Chile y su distribución regional en período 2002-2012, con la finalidad de poder identificar los patrones de la enfermedad que puedan contribuir a establecer políticas y priorizar medidas de intervención.

\section{Material y Método}

Diseño ecológico de base poblacional, fue diseñado para comparar las tasas de mortalidad por CO del país y entre las regiones, así como la distribución de la tasa de mortalidad en Chile por año para el período 2002-2012.

\section{Variables y fuente de datos}

Los datos de mortalidad por cáncer oral fueron obtenido del Subdepartamento de Información Ciudadana (SIAC) del Instituto Nacional de Estadísticas (INE), por la Ley de Transparencia $\mathrm{N}^{\circ}$ 20.285. Información sobre sexo, edad de la muerte (años), localización anatómica bucomaxilofacial (según la Clasificación Internacional de Enfermedades CIE 10, región de residencia y año de muerte por cáncer oral, a partir de los códigos C00-C97 del CIE 10 : labio [C00], lengua [C01-02], encía [C03], piso de boca [C04], paladar [C05], otros sitios mal definidos de la cavidad bucal [C06], glándula parótida [C07], glándulas salivales no especificadas [C08], huesos del cráneo y la cara [C41.0] y hueso maxilar inferior [C41.1]). Se excluyeron del estudio los códigos C09- C10- C11-C12- C13, los cuales corresponden a neoplasias de zona faríngea y código C14 que incluye "Neoplasias malignas de otras partes del labio, la cavidad oral y la faringe" debido a que no se puede separar localización objetivo de este estudio.
La información sobre la población del período 2002-2012 se obtuvo de las Proyecciones de Población del Instituto Nacional de Estadísticas (INE), basado en datos del censo 2002.

\section{Análisis estadístico}

El número total y las proporciones se informan para el total de cáncer oral por año, según ubicación bucomaxilofacial y sexo. La edad se clasificó en diez grupos quinquenales, 0 a $4 ; 5$ a $9 ; 10$ a $14 ; 15$ a $24 ; 25$ a $34 ; 35$ a $44 ; 45$ a $54 ; 55$ a $64 ; 65$ a $74 ; 0-44$, 45-54, 55-64, 65-74 y 75 años y más, para comparación con otras investigaciones y por razones de distribución variable ${ }^{5,7}$. Se calculó la tasa bruta de mortalidad anual y para el período comprendido entre los años 2002 y 2012 en Chile y para cada región. Las tasas se calcularon por 100.000 habitantes, utilizando las estimaciones de población para el país y cada región por año. Las tasas de mortalidad ajustadas por sexo y edad, se calcularon utilizando la población estándar (OMS) y para comparar las tasas regionales también se utilizó a la población chilena proyectada al 30 de junio del 2012. Se utilizó método de estandarización directa, dado que las regiones tienen diferente distribución y la edad tiene una influencia importante en el riesgo de cáncer.

Se calculó razón de tasas para describir la diferencia entre hombres y mujeres por localización anatómica y grupo de edad. Además, se realizó un mapa de las tasas de mortalidad según sexo y un gráfico de tendencias para la descripción de la evolución de la tasa por año.

Para la descripción de la evolución de la tasa por año durante el período estudiado se realizó un gráfico de tendencias. Para la realización del cartograma se usó el software ArcGis versión 10 (Enviromental Systems Research Institute, CA, EE.UU. 2011), la proyección utilizada para cartografiar fue el Datum WGS 1984.

El análisis estadístico se realizó a través de Excel y el software Stata versión 13 (College Station, TX, EE.UU.).

\section{Resultados}

En el período 2002-2012 se registraron 1.611 $(0,67 \%)$ muertes por cáncer oral, de un total de 237.135 muertes por cáncer en Chile. La localización anatómica más frecuente para ambos sexos fue la lengua $(26,5 \%)$, seguido de la glándula parótida $(16,5 \%)$ (Tabla 1$)$. 
El 63,2\% (1.018) de los fallecidos por cáncer oral fueron hombres (H: M, 1.7: 1). La edad mediana fue 67,58 años, siendo la edad mínima 3 años (Región del Biobío) y máxima 104 años (Región de Los Lagos).

La tasa bruta de mortalidad total del período fue 0,89 muertes por 100.000 habitantes $(1,13$ para los hombres, 0,66 para las mujeres). El análisis temporal mostró que las tasas de mortalidad variaron de 0,8 a 1,16, según el año. Para hombres oscilaron entre 0,93 y 1,42 , y para mujeres, las tasas oscilaron entre 0,53 y 0,91 . En el año 2012 se observó un leve incremento, tanto en la población general, hombres y mujeres. Independientemente del sexo, a medida que aumentaba la edad, las tasas de mortalidad también aumentaron, aunque el efecto fue más marcado en el grupo de 75 años y más (Figura 1A).

Tabla 1. Número de muertes por cáncer oral en Chile según sexo y la ubicación anatómica del tumor

\begin{tabular}{|c|c|c|c|c|c|c|c|}
\hline \multirow[t]{2}{*}{ Ubicación Bucomaxilofacial } & \multicolumn{2}{|c|}{ Hombre } & \multicolumn{2}{|c|}{ Mujer } & \multicolumn{2}{|c|}{ Total } & \multirow{2}{*}{$\begin{array}{c}\text { Razón } \\
\text { H:M }\end{array}$} \\
\hline & $\mathbf{n}$ & $\%$ & $\mathbf{n}$ & $\%$ & $\mathbf{n}$ & $\%$ & \\
\hline Labios [COO] & 57 & 3,5 & 22 & 1,3 & 79 & 4,9 & $2.5: 1.0$ \\
\hline Lengua [C01-C02] & 276 & 17,1 & 151 & 9,3 & 427 & 26,5 & 1.8:1.0 \\
\hline Encía [C03] & 10 & 0,6 & 10 & 0,6 & 20 & 1,2 & $1.0: 1.0$ \\
\hline Piso de boca [C04] & 162 & 10,0 & 42 & 2,6 & 204 & 12,6 & $3.8: 1.0$ \\
\hline Paladar [CO5] & 58 & 3,6 & 30 & 1,8 & 88 & 5,4 & $1.9: 1.0$ \\
\hline Otros sitios mal definidos de la cavidad bucal [C06] & 122 & 7,5 & 97 & 6,0 & 219 & 13,5 & $1.2: 1.0$ \\
\hline Gl. Parótida [CO7] & 173 & 10,7 & 92 & 5,7 & 265 & 16,4 & 1.8:1.0 \\
\hline Gl. Salivales no especificadas [CO8] & 40 & 2,4 & 38 & 2,3 & 78 & 4,8 & $1.0: 1.0$ \\
\hline Huesos de la cara [C41.0] & 107 & 6,6 & 102 & 6,3 & 209 & 12,9 & $1.0: 1.0$ \\
\hline Hueso maxilar inferior [C41.1] & 13 & 0,8 & 9 & 0,5 & 22 & 1,3 & $1.4: 1.0$ \\
\hline Total & 1.018 & 63,2 & 593 & 32,8 & 1.611 & 100 & $1.7: 1.0$ \\
\hline
\end{tabular}
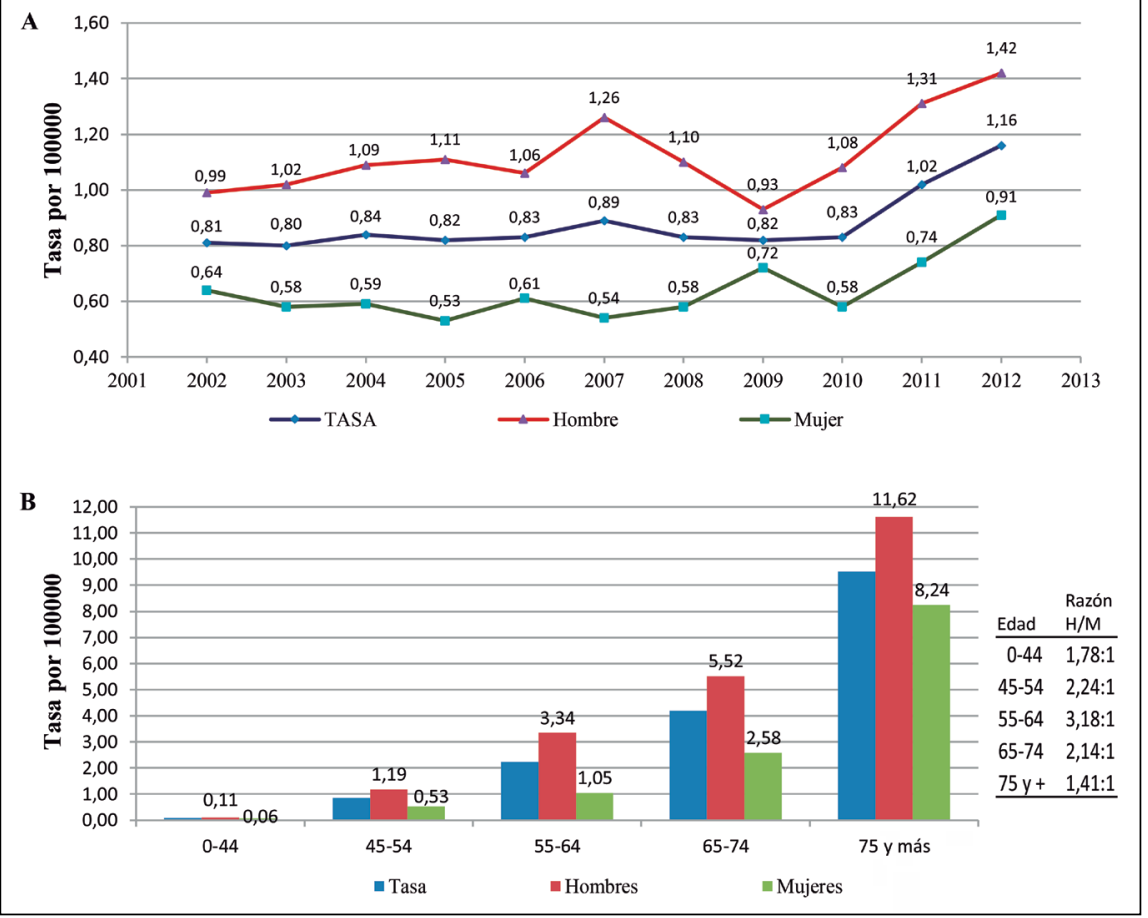

Figura 1. Tasa bruta de mortalidad por cáncer oral según año, sexo y grupo de edad. A. Tendencia de la tasa bruta de mortalidad por cáncer oral según año y sexo Chile 2002-2012. B. Tasa específicas según grupo de edad. Chile 20022012. 
Tabla 2. Tasa bruta y ajustada de mortalidad por edad y sexo para cáncer oral según región. 2002-2012

\begin{tabular}{|c|c|c|c|c|c|c|c|c|c|c|c|c|}
\hline \multirow[t]{2}{*}{ Regiones } & \multirow{2}{*}{$\begin{array}{c}n \\
\text { muertos }\end{array}$} & \multicolumn{2}{|c|}{ Hombres } & \multicolumn{2}{|c|}{ Mujeres } & \multirow[t]{2}{*}{ Población } & \multicolumn{3}{|c|}{ Tasa Específica } & \multicolumn{3}{|c|}{ Tasa Ajustada } \\
\hline & & $\mathbf{n}$ & $\%$ & $\mathbf{n}$ & $\%$ & & H & M & Global & H & M & Global \\
\hline Arica y Parinacota & 20 & 12 & 60,0 & 8 & 40,0 & 2.301 .038 & 1,04 & 0,69 & 0,87 & 1,19 & 0,68 & 0,93 \\
\hline Tarapacá & 27 & 17 & 63,0 & 10 & 37,0 & 3.059 .206 & 1,08 & 0,68 & 0,88 & 1,32 & 0,81 & 1,10 \\
\hline Antofagasta & 71 & 42 & 59,2 & 29 & 40,8 & 6.069 .348 & 1,33 & 1,00 & 1,17 & 1,99 & 1,13 & 1,51 \\
\hline Atacama & 31 & 21 & 67,7 & 10 & 32,3 & 3.078 .347 & 1,33 & 0,67 & 1,01 & 1,55 & 0,69 & 1,12 \\
\hline Coquimbo & 56 & 35 & 62,5 & 21 & 37,5 & 7.453 .558 & 0,95 & 0,56 & 0,75 & 0,85 & 0,50 & 0,75 \\
\hline Valparaíso & 187 & 116 & 62,0 & 71 & 38,0 & 18.523 .418 & 1,27 & 0,75 & 1,01 & 1,27 & 0,56 & 0,89 \\
\hline Metropolitana & 635 & 393 & 61,9 & 242 & 38,1 & 73.210 .520 & 1,10 & 0,65 & 0,87 & 1,28 & 0,57 & 0,88 \\
\hline O’Higgins & 84 & 65 & 77,4 & 19 & 22,6 & 9.370 .530 & 1,38 & 0,41 & 0,90 & 1,45 & 0,38 & 0,90 \\
\hline Maule & 93 & 58 & 62,4 & 35 & 37,6 & 10.792 .298 & 1,08 & 0,65 & 0,86 & 1,13 & 0,59 & 0,84 \\
\hline Biobío & 196 & 129 & 65,8 & 67 & 34,2 & 22.012 .899 & 1,19 & 0,60 & 0,89 & 1,28 & 0,54 & 0,88 \\
\hline Araucanía & 70 & 43 & 61,4 & 27 & 38,6 & 10.305 .385 & 0,84 & 0,52 & 0,68 & 0,89 & 0,46 & 0,65 \\
\hline Los Ríos & 44 & 22 & 50,0 & 22 & 50,0 & 4.198 .953 & 1,19 & 0,91 & 1,05 & 1,22 & 0,79 & 1,00 \\
\hline Los Lago & 66 & 43 & 65,2 & 23 & 34,8 & 8.633 .356 & 0,99 & 0,54 & 0,76 & 1,14 & 0,51 & 0,79 \\
\hline Aysén & 11 & 06 & 54,5 & 05 & 45,5 & 1.098 .947 & 1,04 & 0,96 & 1,00 & 1,30 & 1,14 & 1,22 \\
\hline Magallanes & 20 & 13 & 65,0 & 07 & 35,0 & 1.732 .417 & 1,45 & 0,84 & 1,15 & 1,62 & 0,73 & 1,17 \\
\hline PAIS & 1.611 & 1.018 & 63,2 & 593 & 36,8 & 181.840 .220 & 1,13 & 0,65 & 0,89 & 1,13 & 0,58 & 0,85 \\
\hline
\end{tabular}

El $80,1 \%$ de las muertes ocurrieron a partir de los 55 años y se observó que en los hombres la mortalidad ocurría en edades más tempranas que en las mujeres. En el grupo de edad de 0-44 años, los hombres tuvieron $78 \%$ más muerte por $\mathrm{CO}$ que las mujeres (1.7:1); en el grupo de edad de 45 y 54 años, el número de muertes en los hombres fue más del doble que en mujeres $(2.2: 1)$ y en el grupo de 55 a 64 años fue tres veces más que en las mujeres (3.1:1). A partir de 65 años, la razón empieza a reducir (2.1:1), y en el grupo de edad de 75 años la proporción de muertes por $\mathrm{CO}$ de hombres respecto a las mujeres fue 1,4:1 (Figura 1B).

La tasa de mortalidad ajustada por sexo y edad fue 0,85 muertes por 100.000 habitantes, 1,13 para los hombres y 0,58 para las mujeres. Según la región de residencia, se observó una distribución desigual de las tasas de mortalidad ajustadas por edad y sexo. La mayor tasa de mortalidad ajustada se observó en la Región de Antofagasta (1,51 por 100.000 habitantes) y la Región de Aysén con 1,22, seguido de la Región de Magallanes $(1,17)$. Las tasas más bajas se observaron en la Región de la Araucanía $(0,65)$ y la Región de Los Lagos $(0,79)$.
Respecto al sexo, las tasas ajustadas son poco más elevadas respecto a las tasas específicas (Tabla 2 y Figura 2).

\section{Discusión}

La tasa bruta por cáncer oral en el período estudiado presentó una tendencia a la estabilidad hasta 2010 y un leve incremento desde el año 2011. La localización más frecuente fue la lengua $(26,5 \%)$, seguida de la parótida $(16,4 \%)$. La tasa de mortalidad por cáncer oral en Chile para el período estudiado fue 0,85 por 100.000 habitantes, con diferencias entre las regiones y por sexo. La edad promedio de los muertos por cáncer oral fue 67,58 y fueron los hombres los que mueren más y en edad más temprana que en las mujeres. El leve incremento de la mortalidad se podría explicar por el envejecimiento de la población y la elevada prevalencia de exposición a tabaco y alcohol en Chile. De acuerdo a la localización del cáncer oral, la lengua fue la localización más frecuente; estos resultados son similares a los encontrados por Riera et al. ${ }^{5}$, Zini et al. ${ }^{8}$ y Ordoñez et al. ${ }^{9}$. 


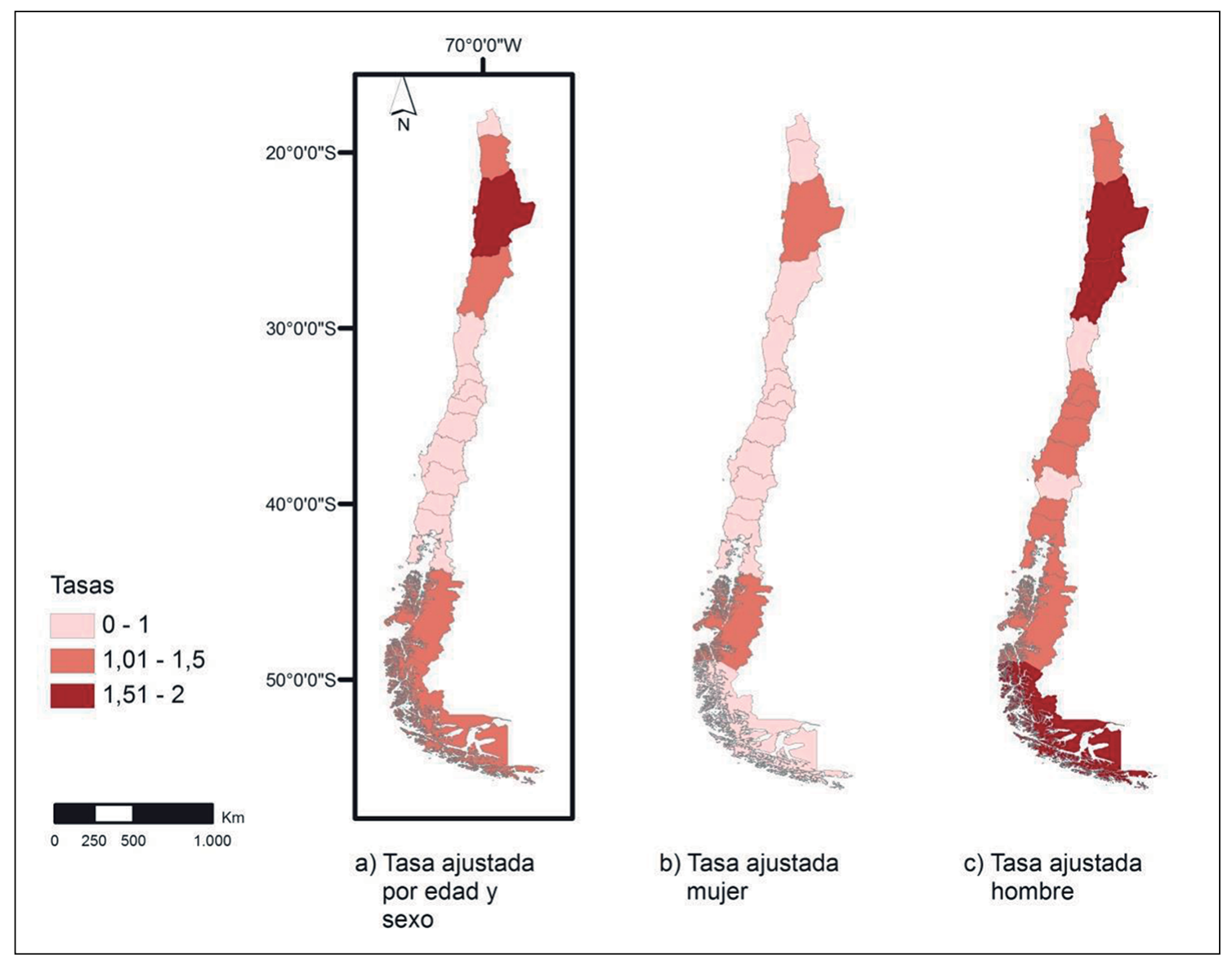

Figura 2. Tasa de mortalidad por cáncer oral por región y sexo, Chile 2002-2012.

La tasa ajustada para Chile es inferior a la reportada por GLOBOCAN para el año 2012 $\left(1,9^{\star} 100.000\right.$ habitantes $)$, más aun si lo comparamos con un país asiático como India $(4,9$ cada 100.000 habitantes) y a los países vecinos de la región como en Uruguay ( 1,9 cada 100.000 habitantes) y Argentina (1,2 cada 100.000 habitantes $)^{2,3}$. Respecto a India, la alta tasa se ha relacionado con el consumo de nuez de betel. Se conoce que su consumo habitual aumenta tres veces el riesgo de desarrollar cáncer oral comparado con los que lo consumen esporádicamente; en Chile se desconoce su consumo ${ }^{10}$. Respecto a Uruguay y Argentina, puede estar relacionada con el consumo de carnes rojas, el cual se ha descrito como factor de riesgo ${ }^{11}$. En Chile, el consumo de carne rojas fue de 24,4 kilos per cápita en 2013, la mitad de lo que se consumió para el mismo año en
Argentina $(59,4)$ y Uruguay $(58,6)^{12,13}$. Otro factor puede ser explicado por el consumo de tabaco, si bien en Chile la prevalencia de consumo de tabaco es $40,6 \%$, según la última encuesta nacional, y se considera el país de mayor consumo a Latinoamérica, el número de cigarrillo en promedio es 10 por día, inferior al promedio por día en Argentina y Uruguay 12 y 15,3 , respectivamente ${ }^{14-16}$. El riesgo de desarrollar un cáncer varía en función de la dosis, duración, calidad y forma de consumo de tabaco $^{14}$. No se descarta una subnotificación de casos por la falta de diagnóstico, relacionado a elevado costo de la atención dental, no cubierta por el sistema sanitario.

La distribución desigual de las tasas de mortalidad según la región de residencia podría ser evidencia de la existencia de otros factores ambientales asociados en algunas áreas de Chile. 
Por ejemplo, en el caso de la zona norte de Chile, especialmente en la Región de Antofagasta, hasta la década 1970-79 la población ha estado expuesta a concentraciones muy altas de arsénico, presentes en el agua de grifo y aire contaminado, siendo un factor importante a tener en cuenta, dado que muchos estudios han reportado un aumento en la mortalidad por cáncer de pulmón y vejiga, junto con otros problemas de salud en la misma área ${ }^{17}$. Aunque hoy en día las concentraciones de arsénico en la zona norte están por debajo de las recomendaciones de la OMS, las consecuencias se siguen observando, especialmente en las enfermedades con largos períodos latentes, como el cáncer ${ }^{18}$. El hecho de que la I Región y en la III Región tengan incidencias más bajas podría apoyar el argumento del arsénico en la Región $\mathrm{II}^{19}$.

En cuanto a la alta tasa de mortalidad por $\mathrm{CO}$ en Aysén, podría explicarse, en parte, por la falta de centros de diagnóstico y tratamiento, al ser una zona extrema del país y escasez de personal sanitario odontólogos y médicos capaces de abordar esta patología ${ }^{20}$.

En este estudio, 63,2\% de los casos ocurrieron en hombres, con una relación hombre: mujer de 1,7:1. Esta razón es menor a lo observado en el estudio de Riera et al. ${ }^{5}$ (H:M: 2,3:1). La diferencia de la razón H:M puede estar asociado al género, porque son las mujeres las que acuden con más frecuencia al médico y suelen estar más informadas sobre sus enfermedades que los hombres ${ }^{21}$. Otro factor es el mayor tiempo exposición al consumo de tabaco y alcohol en hombres respecto a las mujeres. Por otra parte, la disminución de la diferencia entre hombre y mujeres da cuenta que el cáncer oral en mujeres está aumentando, como se ha observado en otras investigaciones, lo que podría relacionarse a que las mujeres están igualando a los hombres en hábitos de tabaco, alcohol y dieta inadecuada en los últimos años ${ }^{22-24}$.

En relación a la edad, la media fue 67,58 años para ambos sexos, similar a la tendencia mundial. El 80\% de los casos de mortalidad se produjeron a partir de los 55 años. Este hallazgo es posible atribuirlo a la acumulación de factores carcinogénicos y a cambios asociados al envejecimiento celular $^{25}$. El cáncer en hombres ocurre en etapas más tempranas que en las mujeres, y fue tres veces más hasta los 64 años acortándose esta razón a partir de los 65 años. Estos datos son similares a los observados a un estudio realizado por la Aso- ciación Americana de Cáncer, en la investigación se reveló que el cáncer oral ocurre en hombres a edades más tempranas (edad media de 40-49 años) que en mujeres (mayores de 65 años) $)^{26,27}$.

Este estudio tiene algunas limitaciones. Una de las principales limitaciones de los datos otorgados por el INE, si bien es una base de datos validada, puede existir sesgo de información generado principalmente en el proceso de registro. No se pudo realizar la descripción de las características histológicas del tipo de cáncer, porque los registros no recogen esa información. Finalmente, el lector debe tener en cuenta la falacia ecológica que surge de los estudios ecológicos. Esto podría conducir a conclusiones erróneas al inferir los hallazgos observados del nivel poblacional al nivel individual.

Como conclusión cabe señalar que las tasas de mortalidad son un indicador para medir la eficacia de programas de prevención de enfermedades y evaluar los efectos o beneficios de diferentes programas de salud en salud pública. Aunque la mortalidad por cáncer oral es menor que la mortalidad por otros cánceres como pulmón, colon y próstata en Chile, no deja de representar un alto costo para el sistema de salud ${ }^{28}$. En el contexto, es esencial el rol del odontólogo en la identificación temprana del tumor o de lesiones potencialmente malignas en la consulta diaria, así como en el diagnóstico y tratamiento precoz, de ahí que autoridades sanitarias podrían potenciar actividades preventivas, especialmente en adultos de 45 años y más, con especial énfasis en los hombres.

Agradecimientos: Los autores agradecen a los Drs. Alfredo Esguep y Kim Kraemer de la Facultad de Odontología Universidad Andrés Bello, por su colaboración en el desarrollo de esta investigación y a la geógrafa Ana Karina Maldonado Alcaíno de la Escuela de Salud Pública de la Universidad de Chile, por su aporte en obtener los resultados del manuscrito.

\section{Referencias}

1. Gupta B, Johnson WW, Kumar N. Global Epidemiology of Head and Neck Cancers: A Continuing Challenge. Oncology 2016; 91 (1): 13-23.

2. World Health Organization. France: International Agency for Research on Cancer; Globocan, Section of Cancer Surveillance [Internet]. 2012. [citado el 10 de 
enero de 2017]. Disponible en: http://globocan.iarc.fr/ Pages/fact_sheets_population.aspx.

3. Ferlay J, Soerjomataram I, Dikshit R, Eser S, Mathers $\mathrm{C}$, Rebelo $\mathrm{M}$, et al. Cancer incidence and mortality worldwide: sources, methods and major patterns in GLOBOCAN 2012. Int J Cancer 2015; 136 (5): 359-86.

4. Santelices Ch MJ, Cárcamo IM, Brenner AC, Montes FR. Cáncer oral en Chile: Revisión de la literatura. Rev Med Chile 2016; 144 (6): 758-66.

5. Riera P, Martínez B. Morbilidad y mortalidad por cáncer oral y faríngeo en Chile. Rev Med Chile 2005; 133 (5): 555-63.

6. Warnakulasuriya S. Global epidemiology of oral and oropharyngeal cancer. Oral Oncol 2009; 45 (4-5): 30916.

7. Ramírez V, Vásquez-Rozas P, Ramírez-Eyraud P. Mortalidad por cáncer oral y faríngeo en Chile, años 20022010. Rev Clin Periodoncia Implantol Rehabil Oral Rev 2015; 8 (2): 133-8.

8. Zini A, Czerninski R, Sgan-Cohen HD. Oral cancer over four decades: epidemiology, trends, histology, and survival by anatomical sites. J Oral Pathol Med 2010; 39(4): 299-305.

9. Ordoñez D, Aragón N, García LS, Collazos P, Bravo LE. Oral cancer in Cali, Colombia: a population-based analysis of incidence and mortality trends. Salud Publica Méx 2014; 56 (5): 465-72.

10. Anand R, Dhingra C, Prasad S, Menon I. Betel nut chewing and its deleterious effects on oral cavity. J Cancer Res Ther 2014; 10 (3): 499-505.

11. Xu J, Yang X, Wu Y, Li X, Bai B. Meat Consumption and Risk of Oral Cavity and Oropharynx Cancer: A Meta-Analysis of Observational Studies. PLoS ONE. 2014; 9 (4): e95048.

12. Matos E, Brandani A. Review on meat consumption and cancer in South America. Mutat Res 2002; 506-507: 243-9.

13. Wünsch-Filho V. The epidemiology of oral and pharynx cancer in Brazil. Oral Oncol 2002; 38 (8): 737-46.

14. MINSAL. Encuesta Nacional de Salud ENS Chile 20092010. [Internet]. 2010 [citado el 2 de febrero de 2017]. Disponible en: http://web.minsal.cl/portal/url/item/ bcb03d7bc28b64dfe040010165012d23.pdf.

15. Secretaría de Programación para la Prevención de la Drogadicción y Lucha contra el Narcotráfico (SEDRONAR). Informe Epidemiologico Sobre el Consumo de Tabaco en Argentina. Disponible en: http://www.observatorio. gov.ar/media/k2/attachments/InformeZEpidemiolgicoZsobreZelZConsumoZdeZTabacoZenZArgentina.
ZAbrilZ2016.pdf. [Consultado el 10 de octubre de 2017].

16. SMU. Tabaquismo en Uruguay. [Internet]. 2014. [citado el 5 de octubre del 2017]. Disponible en: http://www. smu.org.uy/elsmu/comisiones/tabaco/inf_ct_tab_en_ uruguay.pdf.

17. Ferreccio C, Sancha AM. Arsenic exposure and its impact on health in Chile. J Health Popul Nutr 2006; 24: 164-75.

18. World Health Organization. Guidelines for drinking-water quality, third edition, incorporating first addendum. Geneva: WHO, 2006.

19. Castillo H, Venegas G. Impacto y consecuencias del Arsénico en la salud y el medio ambiente en el Norte de Chile. Rev Interamericana Ambiente Tur 2010; 6 (1): 53-60.

20. Goic A. [The Chilean Health Care System: The task ahead]. Rev Med Chile 2015; 143 (6): 774-86.

21. MINSAL, Ministerio de Salud. Gobierno de Chile. Situación Actual de la Salud del Hombre en Chile Disponible en: http://web.minsal.cl/portal/url/item/b7e8f68be82d7f2fe040010165013351.pdf [Citado el 20 de febrero de 2017].

22. Franceschi S, Bidoli E, Herrero R, Muñoz N. Comparison of cancers of the oral cavity and pharynx worldwide: etiological clues. Oral Oncol 2000; 36 (1): 106-15.

23. Bhurgri Y, Bhurgri A, Hussainy AS, Usman A, Faridi $\mathrm{N}$, Malik J, et al. Cancer of the oral cavity and pharynx in Karachi-identification of potential risk factors. Asian Pac J Cancer Prev 2003; 4 (2): 125-30.

24. Saman DM. A review of the epidemiology of oral and pharyngeal carcinoma: update. Head Neck Oncol 2012; 4: 1 .

25. Bettendorf O, Piffkò J, Bànkfalvi A. Prognostic and predictive factors in oral squamous cell cancer: important tools for planning individual therapy? Oral Oncol 2004; 40 (2): 110-9.

26. American Cancer Society. Cancer Statistics Center. Disponible en: https://cancerstatisticscenter.cancer.org/\#!/ cancer-site/Oral\%20cavity\%20and\%20pharynx [Citado el 5 de octubre de 2017].

27. American Cancer Society. Oral Cavity and Oropharyngeal. Disponible en: https:/www.cancer.org/cancer/ oral-cavity-and-oropharyngeal-cancer.html [Consultado el 5 de octubre de 2017].

28. MINSAL, Ministerio de Salud. Gobierno de Chile. Estrategia Nacional de Cáncer Chile 2016. Disponible en: http://web.minsal.cl/wp-content/uploads/2016/10/Estrategia-Nacional-de-Cancer-version-consulta-publica.pdf [Consultado el 5 de octubre de 2017]. 